\title{
Learning of the insoluble conditional reaction problem by rhesus monkeys*
}

\author{
STEVEN F. FLAGG $\dagger$ \\ Washington State University, Pullman, Washington 99163
}

\begin{abstract}
Spiker's modification of the Hull-Spence learning theory predicts that one particular type of conditional reaction problem is insoluble. However, a context-sensitive theory of discrimination learning developed by Medin predicts that this problem is soluble. Eight rhesus monkeys were trained on this previously uninvestigated problem for 36 days. Seven of the eight monkeys learned the problem, thus supporting the context-sensitive theory of discrimination learning.
\end{abstract}

Spence's original learning theory (Spence, 1936, 1937) holds that the effective stimulus in discrimination learning is a complex or compound consisting of several components. A simple two-choice black-white simultaneous discrimination problem has two dimensions, lightness and position, with two values on each dimension. Each effective stimulus is a compound consisting of one value from each dimension, with the resulting four compounds being black-left, white-right, white-left, and black-right. A response to a reinforced stimulus compound results in an increase in the habit strength of each of the components in the compound. The total habit strength of a stimulus compound is equal to the sum of the habits acquired by the components in that compound. The compound containing the consistently reinforced component will acquire more habit strength than any other compound.

Spence's original theory accounted for solution of the simultaneous discrimination problem quite well. However, in 1951, Weise and Bitterman and later Teas and Bitterman (1952) noted that it did not predict learning of successive discrimination problems. In this type of problem, one pair of identical stimuli are presented on some trials and a different pair of identical stimuli on other trials. The $S$ must learn to respond to the left stimulus when confronted with one pair of stimuli and to the right stimulus when confronted with the other pair.

There is no single component that is consistently reinforced in the successive discrimination problem, but this type of problem can be learned by animals in many different species (Reese, 1968). Spence (1952) discussed this dilemma and proposed that, when no single component is consistently rewarded, habit strength is built up to the stimulus compounds rather than to components of the compounds. This two-level theory lacks parsimony, and Spiker $(1963,1970)$ has offered

\footnotetext{
*This investigation, which was the author's MS thesis, was supported in part by United States Public Health Service Grants MH19321 and HD03949 to Roger T. Davis. Appreciation is extended to Douglas L. Medin, Rockefeller University, for his suggestions.

TRequests for reprints should be sent to Steven F. Flagg, Department of Psychology, Washington State University, Pullman, Washington 99163.
}

the principle of stimulus interaction as a simpler, more complete alternative. It states that "... the strength of a habit (or inhibition), occurring to a stimulus component through direct reinforcement (or nonreinforcement) of a compound of which the component is a part, will be reduced when that component appears in a different compound: The amount of the reduction in habit or inhibition strength will be an increasing function of the average dissimilarity between corresponding elements in the two compounds [Spiker, 1970, p. 500]." In other words, the amount of habit strength that generalizes from a component in one compound to a similar component in another compound depends not only on the similarity of these two components but also on the average similarity of the two compounds in which the components appear.

By combining the principle of stimulus interaction with the axioms of Spence's learning theory, Spiker was able to derive equations to predict solutions of several different types of discrimination problems including the simultaneous problem, the successive problem, combinations of successive and simultaneous problems, and even the complex conditional reaction problem.

The conditional reaction problem is shown in Fig. 1. It consists of two simultaneous discrimination problems, Settings 1 and 2 comprising one simultaneous problem and Settings 3 and 4 comprising another. Size is the relevant dimension in each simultaneous problem, but the reward contingencies are reversed from one problem to the other. The dimension signaling this reversal is lightness. The four settings are repeatedly presented to the $S$ in a random order, and the $S$ must learn to respond to the large square if the stimuli are white (Settings 1 and 2) and to the small square if the stimuli are black (Settings 3 and 4). Results from experiments which trained rats (Lashley, 1938), chimpanzees (Nissen, 1951), and children (Hoyt, 1960, 1962) on this problem support Spiker's theory.

However, one type of conditional reaction problem, which is a logical extension of the one in Fig. 1, has not been studied. As can be seen from Fig. 2, this problem consists of two successive discrimination problems, with Settings 1 and 2 comprising one successive problem and 

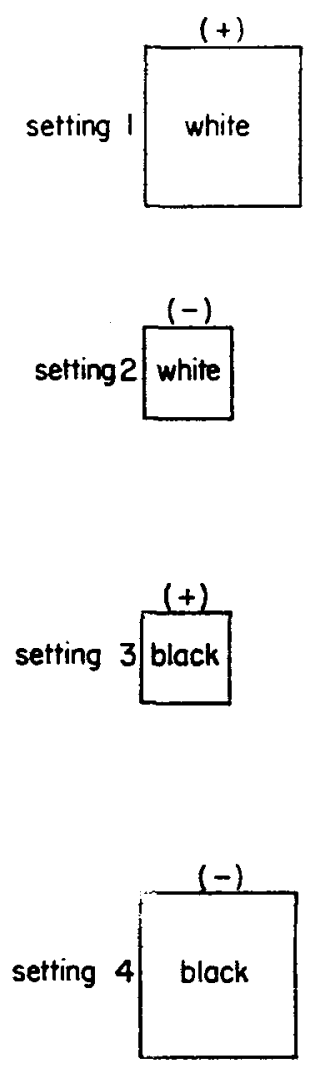

Fig. 1. Conditional reaction discrimination problem with the rewarded stimuli designated by $a+$.

Settings 3 and 4 comprising another successive problem. Size is the relevant dimension in each successive problem, but the reward contingencies are reversed from one problem to the other and lightness signals this reversal. It is also possible to consider Settings 1 and 3 as comprising one successive problem and Settings 2 and 4 the other, with lightness being the relevant dimension in each successive problem and size the reversal cue. The four settings are repeatedly presented to the $S$ in random order. The $S$ must learn to respond to the left stimulus when presented Setting 1 or Setting 4 and to respond to the right stimulus when presented Setting 2 or Setting 3 . Spiker (1970) constructed this problem and found that his theory clearly predicts that the problem cannot be learned.

In contrast to Spiker's prediction, Medin (1973) has developed a context-sensitive theory of discrimination learning which predicts that this type of. conditional reaction problem can be solved. His theory is similar to Spiker's in that both are concerned with generalization decrements due to varying amounts of dissimilarity among the stimuli to be discriminated. Aside from not being formulated from a Hull-Spence point of view, Medin's theory differs from Spiker's in that Spiker used an average dissimilarity measure, whereas Medin used a product rule for combining dissimilarities.

According to Medin's theory, learning consists of acquiring associative information concerning stimuli and the results of responding to those stimuli. The amount of information associated with a stimulus in a given context is reduced by changes either in the stimulus or in its context. The decrement in associative information due to stimulus or context changes along the various dimensions are combined in a multiplicative manner to yield a single similarity measure.

Medin's theory has been successfully applied to many of the problems that Spiker's theory can handle. However, one difference between the theories is that Spiker's theory predicts that the conditional reaction problem depicted in Fig. 2 cannot be learned, while Medin's theory predicts that it can be learned. No experiments with either human or animal Ss had been conducted on this type of conditional reaction problem. The present study tested eight monkeys on this problem in order to determine which prediction is correct.

\section{METHOD}

\section{Subjects}

The Ss were eight thesus monkeys (Macaca mulatta). They ranged in age from 16 to 22 years and for the last 14 years had nearly identical training on experiments dealing with learning, memory, and perception.

\section{Apparatus}

The stimuli were four large squares $(10 \times 10 \mathrm{~cm})$ and four small squares $(5 \times 5 \mathrm{~cm})$ cut from $1 / 8$-in. pressboard. Two of the large squares and two of the small squares were painted flat black. The other four squares were painted flat white.

The monkeys were tested in a Wisconsin General Test Apparatus (WGTA, Harlow, 1949) with the formboard painted flat gray and slanted $50 \mathrm{deg}$ from the horizontal. One stimulus convered each of the two foodwells, which were $8 \mathrm{in}$. apart center to center. Monkeys responded by displacing one of the two stimuli, and their response was reinforced with a raisin in the foodwell if the correct stimulus was chosen.
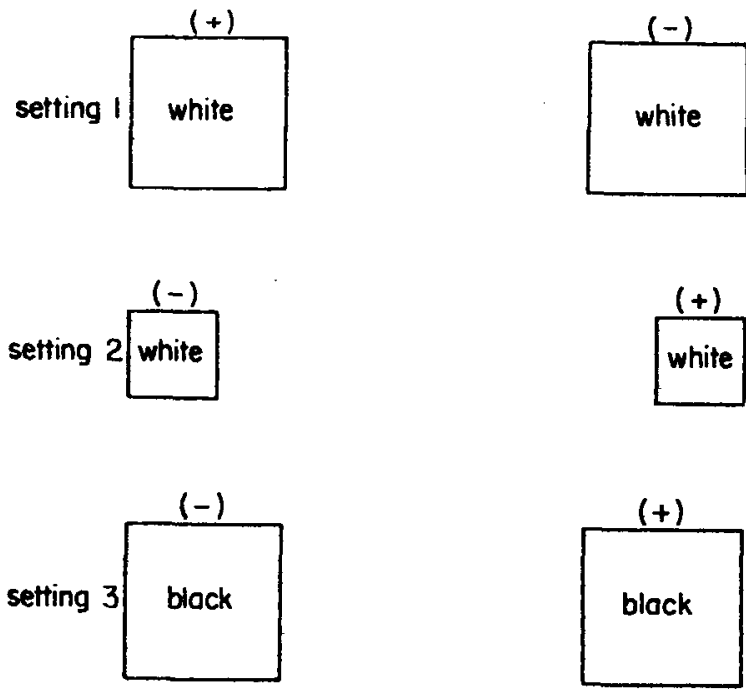

$(+)$
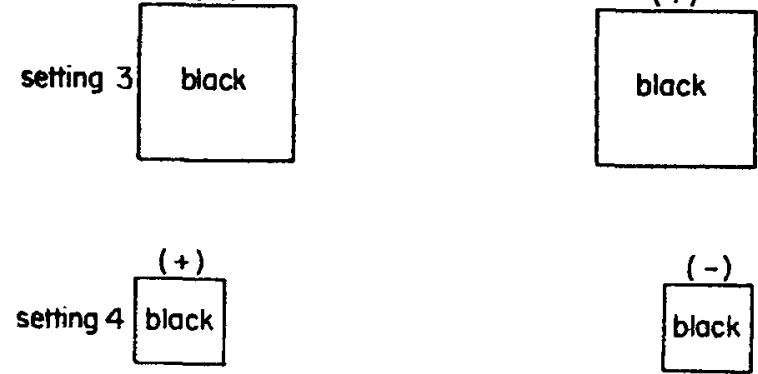

Fig. 2. Conditional reaction discrimination problem tested in this experiment with the rewarded stimuli designated by $a+$ 


\section{Procedures}

The monkeys were trained for 36 days on the problem shown in Fig. 2. Half of the animals were required to learn the problem exactly as depicted in Fig. 2 . The other four animals had the same problem, but the correct stimulus in each setting was the opposite of what it is in the figure.

Each animal received the problem 10 times a day, for a total of 40 trials per day. The order of presenting the settings was randomized except that the entire problem was presented before any part of it was repeated. A noncorrection procedure was used in which correct response always resulted in a raisin reinforcement. The dependent variable was the percentage of correct responses. The learning criterion for each $\mathrm{S}$ was set at $80 \%$ correct responses on each of the four settings in a single day in order to insure that all aspects of the problem were learned. This criterion was arbitrarily selected before the experiment as a very stringent criterion of learning. The probability of a $S$ meeting this criterion by chance is .00000256 .

\section{RESULTS}

For the purpose of analysis, the 36 days of training were broken into nine blocks of 4 days each (practice). The only other term analyzed for within-Ss variance was problem setting. An analysis of variance showed that the effect of practice was significant $(F=39.17$, $\mathrm{df}=8 / 56$, $\mathrm{p}<.001)$. Neither the effect of problem setting $(\mathrm{F}<1)$ nor the Practice by Problem Setting interaction $(F<1)$ was significant.

The significant effect of practice on percentage of correct responses can be seen clearly in Fig. 3. Mean percent correct responses increased from $52 \%$ on Day 1 to $86 \%$ on Day 36 . A t test indicated that performance on Day 36 was significantly above the chance level of $50 \%(\mathrm{t}=8.95, \mathrm{df}=7, \mathrm{p}<.001)$.

All monkeys except one met the learning criterion of $80 \%$ correct responses on each of the four settings at least five times during the 36 days of the experiment. The mean number of times these seven monkeys met the criterion was 11 .

The mean number of days before the criterion was met was 15.9 days, with the first monkey meeting it on Day 6 and the last monkey meeting it on Day 27 (excluding the one monkey that never met the criterion). Thus, seven of the eight monkeys did indeed learn the entire problem. The one monkey that never met the learning criterion had a mean of $70 \%$ correct responses for the last 10 days of the experiment. On 11 different days during the experiment, her performance was between $75 \%$ and $80 \%$ correct, indicating improvement even though she never quite met the stringent learning criterion.

\section{DISCUSSION}

Contrary to Spiker's (1970) prediction, animals can solve the conditional reaction problem depicted in Fig. 2. It must be concluded that neither independent summation of component habit strengths (Spence, 1936, 1937) nor Spiker's stimulus interaction hypothesis (Spiker, 1963, 1970) offer a completely adequate account of animal discrimination learning.

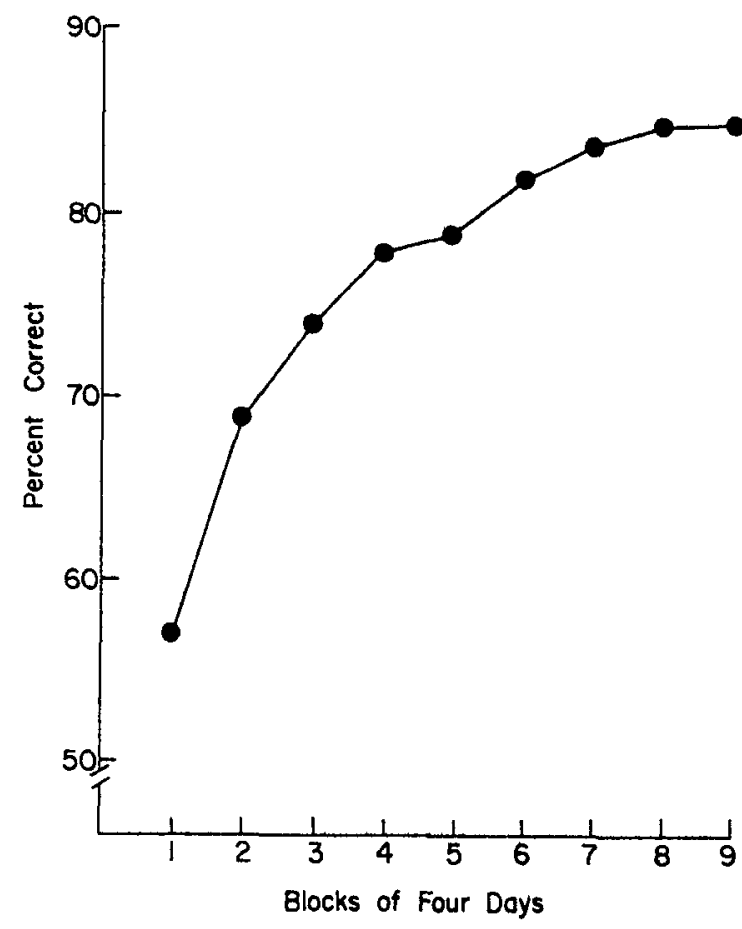

Fig. 3. Percent correct responses as a function of practice.

It would be possible to account for the present data by assuming, as Spence (1952) did, that in certain situations habit is acquired by the entire rewarded compound rather than by its components. However, this formulation is inadequate because Spence did not include a rule specifying the amount of generalization from one compound to other compounds. In addition, it results in a two-level theory of discrimination learning, and one is still left with the task of specifying when each process comes into play.

A more parsimonious explanation of the present results can be found in the context-sensitive theory of discrimination learning proposed by Medin (1973). Whatever the outcome of a trial on Setting 1 in Fig. 2, information will be associated with both stimuli. If we let $R$ represent the information associated with the left square due to a response to it and $\mathrm{p}(0<\mathrm{p}<1)$ represent the generalization of associative information due to the position similarity, then $\mathrm{pR}$ will be associated with the right square. The difference in information associated with each stimulus as a result of such a trial is R-pR or $(1-p) R$.

Similarly, if the $\mathrm{S}$ responds to the right square, nonreward $(\mathrm{N})$ will be associated with that stimulus. Nonreward will also be associated with the left square, but to a lesser degree because it differs in position. Thus, the difference in information due to such a trial is $(1-p) N$. The effective information gain in Setting 1 from either type of trial is $1-p$.

Now consider what happens to the difference in associative information for Setting 1 when the $\mathrm{S}$ makes a response to Setting 2 . If the $\mathrm{S}$ responds to the right square, reward is associated with that stimulus and also, 
to a lesser degree, with both stimuli in Setting 1 . The information associated with the left stimulus in Setting 1 is decreased because it differs in size and position from the right stimulus in Setting 2. The information associated with the right stimulus in Setting 1 due to a response to the right stimulus in Setting 2 is decreased because of the difference in size. Similarly, if the $S$ responds to the left stimulus in Setting 2, nonreward is associated with both stimuli in Setting 1 , but to a lesser degree because they differ in size and position from the left stimulus in Setting 2. In either case, if we let $s$ $(0<s<1)$ represent the generalization due to the size similarity, the net information gain for Setting 1 due to a response in Setting 2 is sp-s.

The effect on the associative information of Setting 1 due to responding in Settings 3 and 4 can be calculated in a similar manner. The net information gain in Setting 1 due to responding in Setting 3 is pb-b (b represents the generalization due to the lightness similarity, such that $0<b<1$ ) and that due to Setting 4 is bs-psb.

The total information gain in Setting 1 is the sum of the gains realized in each setting, or $1-p+s p-s+p b-b+b s-p s b$, which factors into $(1-p)(1-s)(1-b)$. This value represents the total information gain for any of the four settings of the problem. The total information gain for the problem in Fig. 2 is greater than zero because $p, s$, and $b$ are less than one due to the differences in position, size, and lightness among the various stimuli. Although no gain in information implies an insoluble problem, a net gain in information does not in itself imply a soluble problem. A performance rule of some kind is necessary before one can predict whether or not the net gain in available information will lead to solution of the problem.

Medin (1973) performed a computer simulation on the present problem employing the assumptions of his context-sensitive theory in conjunction with Estes's $(1962,1966)$ feedback or scanning model and found that this theory predicts the problem can be learned.

In summary, neither Spence's theory $(1936,1952)$ nor Spiker's (1970) principle of stimulus interaction can adequately account for the present data. In contrast, Medin's (1973) context-sensitive theory of discrimination learning is compatible with the present data.

\section{REFERENCES}

Estes, W. W. Theoretical treatments of differential reward in multiple-choice learning and two person interactions. In J. H. Criswell, H. Solomon, and P. Suppes (Eds.), Mathematical methods in small group process Stanford, Calif: Stanford University Press, 1962. Pp. 133-149.

Estes, W. K. Transfer of verbal discriminations based on differential reward magnitudes. Journal of Experimental Psychology, 1966, 72, 276-283.

Harlow, H, F. The formation of learning sets. Psychological Review, 1949, 6, 51-65.

Hoyt, J. M. Effect of similarity of reversal cues on learning of successive stimuhs reversals in children. Unpublished master's thesis, University of Iowa, 1960. (Briefly cited in Spiker, C. C. An extension of Hull-Spence discrimination learning theory. Psychological Review, 1970, 77, 496-515.)

Hoyt, J. M. Serial reversal and conditioned discrimination learning in children. Unpublished doctoral dissertation, University of Iowa, 1962. (Briefly cited in Spiker, C. C. An extension of Hull-Spence discrimination learning theory. Psychological Review, 1970, 77, 496-515.)

Lashley, $K$. S. Conditional reactions in the rat. Journal of Psy chology, 1938, 6, 311-324.

Medin, D. L. A context-sensitive theory of discrimination learning. Paper presented at a conference on discrimination learning models, Rockefeller University, New York, 1973.

Nissen, H. W. Analysis of a complex conditional reaction in chimpanzee. Journal of Comparative \& Physiological Psychology, 1951, 44, 9-16.

Reese, H. W. The perception of stimulus relations. New York: Academic Press, 1968.

Spence, $K$. W. The nature of discrimination learning in animals. Psychological Review, 1936, 43, 427-499.

Spence, $K$. W. The differential response in animals to stimuli varying within a single dimension. Psychological Review, $1937,44,430-444$.

Spence, $K$. W. The nature of the response in discrimination learning. Psychological Review, 1952, 59, 89-93.

Spiker, C. C. The hypothesis of stimulus interaction and an explanation of stimulus compounding. In L. P. Lipsitt and C. C. Spiker (Eds.), Advances in child development and behavior. Vol. 1. New York: Academic Press, 1963.

Spiker, C. C. An extension of Hull-Spence discrimination learning theory. Psychological Review, 1970, 77, 496-515.

Teas, D. C., \& Bitterman, M. E. Perceptual organization in the rat. Psy chological Review, 1952, 59, 130-140.

Weise, P., \& Bitterman, M. E. Response-selection in discrimination learning. Psychological Review, 1951, 58, 185-195.

(Received for publication Novem ber 29, 1973; revision accepted April 2, 1974.) 